\title{
Silencing of the ARK5 gene reverses the drug resistance of multidrug-resistant SGC7901/DDP gastric cancer cells
}

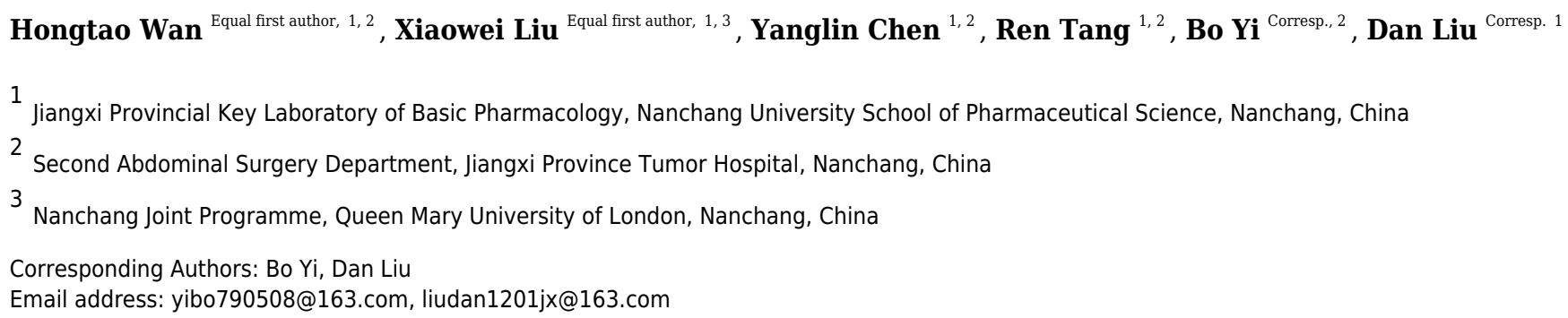

For several years, the multidrug resistance (MDR) of gastric cancer cells has been a thorny issue worldwide regarding the chemotherapy process and needs to be solved. Here, we report that the ARK5 gene could promote the multidrug resistance of gastric cancer cells in vitro and in vivo. In this study, LV-ARK5-RNAi lentivirus was used to transfect the parental cell line SGC7901 and MDR cell line SGC7901/DDP to construct a stable model of ARK5 interference. Subsequently, the cells were treated with four chemotherapeutic drugs, cisplatin (DDP), adriamycin (ADR), 5-fluorouracil (5-FU) and docetaxel (DR) and were subjected to the CCK8, colony formation, adriamycin accumulation and retention, cell apoptosis and other assays. The study found that, in vitro, the expression of ARK5 in MDR gastric cancer cells was significantly higher than that in parental cells. Additionally, when treated with different chemotherapeutic drugs, compared with parental cells, MDR cells also had a higher cell survival rate, higher colony formation number, higher drug pump rate, and lower cell apoptosis rate. Additionally, in xenograft mouse models, MDR cells with high ARK5 expression showed higher resistance to chemotherapeutic drugs than parental cells. Overall, this study revealed that silencing the ARK5 gene can effectively reverse the drug resistance of MDR gastric cancer cells to chemotherapeutic drugs, providing insights into the mechanism of this process related to its inhibition of the active pump-out ability of MDR cells. 

resistant SGC7901/DDP gastric cancer cells

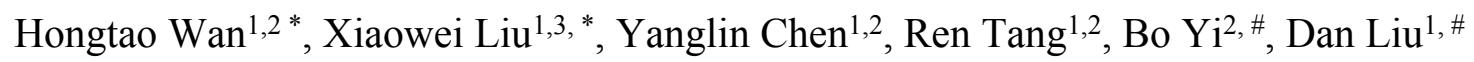

1Jiangxi Provincial Key Laboratory of Basic Pharmacology, Nanchang University School of Pharmaceutical Science, Nanchang 330006, P.R.China

${ }^{2}$ Second Abdominal Surgery Department, Jiangxi Province Tumor Hospital, Nanchang 330029, P.R. China

${ }^{3}$ Nanchang Joint Programme, Queen Mary University of London, Nanchang 330006, P.R. China

*These authors contributed equally to this work.

\#Corresponding author:

Bo Yi, PhD

Jiangxi Province Tumor Hospital

Nanchang 330029, PR China

E-mail: yibo790508@163.com

Dan Liu, PhD

Jiangxi Provincial Key Laboratory of Basic Pharmacology

Nanchang University School of Pharmaceutical Science

Nanchang 330006, PR China

E-mail: liudan1201jx@163.com

\begin{abstract}
For several years, the multidrug resistance (MDR) of gastric cancer cells has been a thorny issue worldwide regarding the chemotherapy process and needs to be solved. Here, we report that the ARK5 gene could promote the multidrug resistance of gastric cancer cells in vitro and in vivo. In this study, LVARK5-RNAi lentivirus was used to transfect the parental cell line SGC7901 and MDR cell line SGC7901/DDP to construct a stable model of ARK5 interference. Subsequently, the cells were treated with four chemotherapeutic drugs, cisplatin (DDP), adriamycin (ADR), 5-fluorouracil (5-FU) and
\end{abstract}


docetaxel (DR) and were subjected to the CCK8, colony formation, adriamycin accumulation and retention, cell apoptosis and other assays. The study found that, in vitro, the expression of ARK5 in MDR gastric cancer cells was significantly higher than that in parental cells. Additionally, when treated with different chemotherapeutic drugs, compared with parental cells, MDR cells also had a higher cell survival rate, higher colony formation number, higher drug pump rate, and lower cell apoptosis rate. Additionally, in xenograft mouse models, MDR cells with high ARK5 expression showed higher resistance to chemotherapeutic drugs than parental cells. Overall, this study revealed that silencing the ARK5 gene can effectively reverse the drug resistance of MDR gastric cancer cells to chemotherapeutic drugs, providing insights into the mechanism of this process related to its inhibition of the active pump-out ability of MDR cells.

\section{Introduction}

Gastric cancer as a lethal malignancy ranking fifth among cancers in humans, while ranking third in tumor-related mortality worldwide[1]. Approximately one million people are diagnosed with gastric cancer every year, among whom approximately seven hundred thousand die from the disease[2, 3]. Thus, the current situation of gastric cancer remains severe.

In recent years, the preferred treatment for gastric cancer is still chemotherapy and surgery, but neither has effectively improved the survival rate[4,5] while the five-year survival rate is approximately $20 \%$ presently[6,7]. Additionally, chemotherapy resistance plays a crucial role in the failure of its treatment. Initially, tumors generally respond to chemotherapy, but most of the time they eventually recur[8] and develop varying levels of cross-resistance to a series of chemotherapeutic drugs after exposure to one drug, and this phenomenon is known as multidrug resistance (MDR) $[9,10]$. Therefore, to improve the sensitivity of tumors to drugs has become a difficult problem in the clinical treatment of gastric cancer while providing an effective entry point for our research.

Studies have demonstrated that tumor cells mainly rely on mechanisms mediated by an efflux pump Pglycoprotein (P-gp) to reduce the accumulation of drugs within cells and alter the subcellular distribution of toxic substances to produce multidrug resistance[11, 12]. As a member of the ABC (ATP-binding cassette) family[13-15], the P-gp is reported to be highly expressed in cancer cells[16] and is regarded as the main source of multidrug resistance. However, the latest study found that downregulation of the expression of $\mathrm{ABC}$ transporter cannot completely reverse the multidrug resistance of tumor cells, such as gastric cancer cells[17]. Although P-gp is highly expressed in some gastric cancer patients, it was not related to the poor prognosis of patients who were treated with adriamycin and 5-fluorouracil therapy[18, 19]. Moreover, in a previous study comprising fifty samples of gastric cancer patients, P-gp could only be detected in $10 \%$ of them[20]. Thus, some other important pathways exist regarding the occurrence of tumor drug resistance.

Previous studies have indicated that AMP-activated protein kinase (AMPK) is a sensor of metabolism and a regulator of energy homeostasis [21] and has positive effects on promoting gastric cancer[22]. ARK5, a member of the AMPK family, has been suggested to be closely related to cell survival and cell function of tumors[23]. Moreover, the relationships between ARK5 and cancer cells have been verified in many cancer types-for instance, cholangiocarcinoma[24], hepatoma[25] and nasopharyngeal carcinoma[26]. The upstream mediator of ARK5 has been identified as AKT[27], LBK1[23], CaMKKb[28] and TAK1[29]. 
It has been proposed that AKT phosphorylation could stimulate intrusion activity through activating ARK5 to inhibit apoptosis. Meanwhile, ARK5 silencing causes overactivation of the downstream target mTOR, eventually leading to cell death[30].

According to recent literature, ARK5 was confirmed to be associated with drug resistance in multiple tumors. For instance, the downregulation of ARK5 could significantly increase the chemical sensitivity of lung cancer cells to cisplatin[31]. Hepatic cancer cells with high expression of ARK5 showed higher resistance to adriamycin than that of cells with low expression of ARK5[25]. Similar results have been shown in cases of cholangiocarcinoma[24]. Thus, we propose that ARK5 plays a key role in the development of drug resistance in tumors. Additionally, ARK5 has been discovered to be closely related to the metastasis and deterioration of gastric cancer cells[32]. However no relevant literature has confirmed the role of ARK5 in the mechanism of multidrug resistance of gastric cancer.

To investigate the molecular basis of the multidrug resistance of gastric cancer, LV-ARK5-RNAi lentivirus was used to transfect the parental cells SGC7901 and MDR cells SGC7901/DDP to construct a stable model of ARK5 interference. Subsequently, the cells were treated with four chemotherapeutic drugs - cisplatin (DDP), adriamycin (ADR), 5-fluorouracil (5-FU) and docetaxel (DR) - and the CCK8, colony formation, adriamycin accumulation and retention, and cell apoptosis assays were performed.. We also generated another in vivo xenograft mouse model to explore the relationship between ARK5 and multidrug resistance of gastric cancer cells, and whether interference with the expression of ARK5 protein could effectively reverse the drug resistance of SGC7901/DDP.

\section{Materials and methods}

\subsection{Cell culture}

The human parental gastric cancer cell line SGC7901 was purchased from the Cell Bank of the Chinese Academy of Sciences (Shanghai, China), and cisplatin-induced multidrug-resistant gastric cancer cell lines SGC7901/DDP were purchased from XiangYa School of Medicine, CSU. The cells were cultured in plates containing the culture medium RPMI-1640 (Solarbio, Beijing, China) with 10\% fetal bovine serum (HyClone, America) and double-antibody penicillin-streptomycin. All the cells were grown in a humidified incubator at a constant temperature of 37 degrees with $5 \%$ carbon dioxide.

\subsection{Transfection}

The LV-ARK5-RNAi lentiviruses and negative control (NC) lentiviruses were constructed by Genechem (Shanghai, China). The multidrug-resistant gastric cancer cell line SGC7901/DDP was seeded on 6-well plates and cultured with LV-ARK5-RNAi within the RPMI-1640+HiTransG A transfection system, and then the medium was changed after $12 \mathrm{~h}$ followed by incubation for $72 \mathrm{~h}$ to achieve optimal interference efficiency for follow-up experiments.

\section{3 western blot analysis}

The RIPA lysate (Sigma, America) and PMSF (Sigma, America) were used to lyse and extract proteins at a ratio of 100:1. The concentration of the centrifuged supernatant was measured using a BCA kit (Beyotime, China), and then the protein fluid was mixed with loading buffer (Solarbio, Beijing, China) 
and denatured at $100{ }^{\circ} \mathrm{C}$ for $5 \mathrm{~min}$. The extracted lysates were separated by $10 \%$ SDS-PAGE and transferred onto PVDF membrane. Next, the membranes were incubated with primary antibody ( $\beta$-actin from Abcam, America; ARK5 form Cell Signaling Technology and Santa Cruz Biotechnology, America)] overnight and then were incubated with secondary antibody (anti-rabbit IgG/anti-mouse IgG, ZSGB-Bio, China) for $1.5 \mathrm{~h}$. Finally, protein expression can be observed by chemiluminescence and analyzed using Image lab.

\subsection{Cell viability assay}

The cell viability was gauged using Cell Counting Kit-8 (TransGen Biotech, Beijing, China). The assays were performed on four different cell groups - parental gastric cancer cells (SGC7901), multidrugresistant gastric cancer cells (SGC7901/DDP), lentiviruses-transfected multidrug-resistant gastric cancer cells (SGC7901/DDP-shARK5), and negative lentivirus-transfected, multidrug-resistant gastric cancer cells (SGC7901/DDP-NC). All cell groups were treated with cisplatin (DDP), 5-fluorouracil (5-Fu), adriamycin (ADR) and docetaxel (DR) (the drugs were purchased from Macklin, Shanghai, China), and ten groups were set up including the blank group, negative group, and 6 experimental groups with different concentrations of the corresponding drug. After reviewing the literature, we found that the plasma peak concentration (PSC) is a common index in cancer chemotherapy [33-35], and a previous study [36] had successfully applied the plasma peak concentration of four drugs to the cell viability assay of gastric cancer cells. In particular, the four chemotherapeutic drugs were added to the experimental cells of each group at $0.25,0.5,1,2,4$ and 8 times their PSC, and each concentration was repeated in 6 wells. Additionally, the peak serum concentrations of DDP, 5-FU, ADR and DR in the human body are 2.0 $\mu \mathrm{g} / \mathrm{ml}, 10 \mu \mathrm{g} / \mathrm{ml}, 0.4 \mu \mathrm{g} / \mathrm{ml}$ and $0.04 \mu \mathrm{g} / \mathrm{ml}$, respectively.

First, cells of different groups were seeded into 96-well-plates and cultured for $24 \mathrm{~h}$ to grow adherently. On the second day, the SGC7901/DDP-shARK5 and SGC7901/DDP-NC groups were treated with lentiviruses. Next, four drugs were added to the corresponding experimental group according to different concentration gradients, followed by culture for $48 \mathrm{~h} 48 \mathrm{~h}$ [37]. Thereafter, CCK-8 reagent was added to each well according to the manufacturer's instructions followed by incubation for $2 \mathrm{~h}$ away from light. The absorbance was then measured at a wavelength of $450 \mathrm{~nm}$ (A450) using a microplate reader (Thermo Scientific, America); the OD measurements were repeated three times and the average was recorded.

\subsection{Apoptosis assay}

The cells of each experimental group were treated with DDP, 5-FU, ADR and DR according to their corresponding peak serum concentrations and the apoptosis rate was evaluated for each group. First, each group was digested with trypsin (Solarbio, America) without EDTA and then was transferred to a brown EP tube after resuspension and centrifugation twice. After discarding the supernatant, $500 \mu$ l of Annexin binding solution was added to each EP tube to suspend the cells, and $5 \mu \mathrm{l}$ of Annexin V-FITC dye (BestBio, Shanghai, China) was added to the binding solution. After mixing, the cells were incubated at $4^{\circ} \mathrm{C}$ for $15 \mathrm{~min}$ in the dark, and then $10 \mu \mathrm{l}$ of PI dye (BestBio, Shanghai, China) was added and incubated for $5 \mathrm{~min}$ in the dark. Finally, the apoptotic cells were quantified by flow cytometry (Beckman, America).

\subsection{Colony formation assay}


151 The rate of colony formation is the inoculation survival rate of cells and can reflect the proliferation

152

153

154

155

156

157

158

159

160

161

162

163

164

165

166

167

168

169

170

171

172

173

174

175

176

177

178

179

180

181

182

183

184

185

186

187

188

189

190

191

ability of the cell population. First, 800 cells per well were inoculated into the six-well plate and were cultured at $37^{\circ} \mathrm{C}$ for $24 \mathrm{~h}$. Next, four anti-tumor drugs were added to each experimental group according to their peak serum concentrations and then were cultured for 2 to 3 weeks. When a cell clone visible to the naked eye appeared on the plates, the culture was stopped. After washing and drying, the cell was fixed with $4 \%$ polymethanol for $15 \mathrm{~min}$. Subsequently, the cells were dyed with $0.1 \%$ crystal violet for 20 min, the dye was washed off, and the cells were dried in air. Finally, the colony numbers of more than 50 cells were counted under a microscope, and the colony formation rates were calculated according to the following formula: (Colony formation rate $=$ number of colonies/inoculated cells $\times 100 \%$ ).

\subsection{In vitro adriamycin accumulation and retention assay}

Adriamycin (ADR) is a drug whose fluorescence could reflect the relative content of the drug in cells, indirectly reflecting the ability of the cells to actively pump out chemotherapeutic drugs. First, the cells of each experimental group were seeded into 6 -well plates with $5 \times 10^{6}$ cells per well. Next, according to the literature[36, 38], $5 \mu \mathrm{g} / \mathrm{ml}$ of fluorescent ADR was added to the culture medium of the ADR accumulation group and ADR retention group, while no drug was added to the control group, followed by incubation for $1 \mathrm{~h}$. Specifically, the ADR retention group was washed three times with PBS, followed by incubation with conventional 1640 culture medium for $1 \mathrm{~h}$. Subsequently, the fluorescence of ADR in cells was detected by flow cytometry with an excitation light wavelength of $488 \mathrm{~nm}$ and an absorption light wavelength of $575 \mathrm{~nm}$. The pump rate of ADR = (intracellular ADR accumulation - intracellular ADR retention)/(intracellular ADR accumulation) $\times 100 \%$.

\subsection{Xenograft mouse models}

Ten 6-week-old female NOD/SCID mice weighing approximately $20 \mathrm{~g}$ were purchased from Hunan SJA Laboratory Animal Co., Ltd. and were randomly divided into two groups, five in each group. The mice were kept in SPF animal rooms with five mice in each cage in Nanchang Royo Biotech Co., Ltd. The constant temperature of the environment was $22^{\circ} \mathrm{C}$ to $25^{\circ} \mathrm{C}$, and the humidity was approximately $40 \%$. Additionally, the mice were fed with appropriate irradiated feed and sterile water.

5-FU has been widely used as a conventional chemotherapeutic agent for the clinical treatment of gastric cancer[39]. Therefore, we selected 5-FU, a representative chemotherapeutic drug, to further verify whether the drug resistance of gastric cancer cell lines with high ARK5 expression was higher than that of parental cell lines in vivo. First, during the experiment, SGC7901 cells and SGC7901/DDP cells were digested with trypsin and then were resuspended at $2 \times 10^{7}$ cells $/ \mathrm{ml}$ with culture medium. Additionally, the mice were anesthetized with $1 \%$ pentobarbital sodium $(0.1 \mathrm{ml} / 20 \mathrm{~g})$ and each was inoculated subcutaneously with $2 \times 10^{6}$ cells in $100 \mu \mathrm{l}$ of medium. On the 10th day postinjection, small lumps of the same size could be observed on each mouse, confirming successful inoculation. Thereafter, mice in both the SGC7901 and SGC7901/DDP groups were intraperitoneally injected with anesthetic pentobarbital sodium and $20 \mathrm{mg} / \mathrm{kg}$ of 5-FU twice a week for three weeks. Particularly, the weight and tumor size of mice were measured with a sterile Vernier caliper every three days, and the volumes of tumors were calculated using the following formula: Volume $=\left(\right.$ length $\times$ width $\left.{ }^{2}\right) / 2[40]$, where the length and width refer to the maximum and minimum diameters, respectively. On the 31 st day postinjection, the mice were

Peer] reviewing PDF | (2020:02:45845:1:0:CHECK 14 May 2020) 
192

193

194

195

196

197

198

199

200

201

202

203

204

205

206

207

208

209

210

211

212

213

214

215

216

217

218

219

220

221

222

223

224

225

226

227

228

229

230

231

232

weighed for the last time, and then were anesthetized with pentobarbital sodium and sacrificed by breaking the neck. Finally, the tumors were separated from the subcutaneous region of the mice with sterile surgical instruments, and the weight and volume were measured.

All animal procedures were approved by the Laboratory Animal Ethics Committee of Nanchang Royo Biotech Co., Ltd. (Nanchang, China; approval number: RYE2018081801), and all laboratory mice were treated strictly according to the Institutional Animal Care's guidelines in the Guide for the Care and Use of Laboratory Animals published by the US National Institutes of Health (NIH Publication No. 85-23, revised 1996).

\subsection{Statistical analysis}

The mean \pm SEM was used for the data processing of each experimental group. Graphpad Prism 6.0 statistical software was used to analyze the homogeneity test of variance, one-way ANOVA, and least significant difference (LSD). P values less than 0.05 were considered to be statistically significant.

\section{Results}

3.1 The ARK5 protein in multidrug-resistant SGC7901/DDP cells is highly expressed.

To investigate the differences in the expression levels of ARK5 protein between parental SGC7901 gastric cancer cells and multidrug-resistant SGC7901/DDP gastric cancer cells, western blot analysis was performed. Compared with the parental cell line SGC7901, the expression level of ARK5 in cisplatininduced multidrug-resistant cell line SGC7901/DDP was significantly upregulated (Fig. 1).

\subsection{Interference efficiency of LV-ARK5-RNAi}

After the transfection of multidrug-resistant SGC7901/DDP cells with positive and negative shARK5 lentiviruses, the expression of ARK5 in each group was detected by western blotting. The analysis showed that, compared with multidrug-resistant cells without lentivirus transfection, the expression of ARK5 protein in SGC7901/DDP-shARK5 cells transfected with positive lentiviruses was significantly decreased, while that in SGC7901/DDP-NC cells transfected with negative lentiviruses was unchanged (Fig. 2). This result indicated that this lentivirus transfection system can be used in subsequent experiments.

\subsection{Silencing of the ARK5 gene in MDR SGC7901/DDP cells reduces the viability of cells following chemotherapeutic drug treatment}

The CCK-8 assay was used to explore the relationship between the ARK5 gene and multidrug-resistant gastric cancer cells. After chemotherapeutic drug treatment, the survival rate of multidrug-resistant SGC7901/DDP cells with high ARK5 expression was significantly higher than that of parental SGC7901 with low ARK5 expression (Fig. 3). However, after the ARK5 gene was silenced by shRNA-ARK5, the survival rate of multidrug-resistant cells was significantly decreased compared with that of the normal SGC7901/DDP cells. Additionally, when the transfected lentivirus was negative, no significant change was observed in the survival rate. Meanwhile, the value of $\mathrm{IC}_{50}$ (Table 1), which indicates the drug sensitivity of cells, was lower in SGC7901/DDP-shARK5 cells than in normal SGC7901/DDP cells. 
233

234

235

236

237

238

239

240

241

242

243

244

245

246

247

248

249

250

251

252

253

254

255

256

257

258

259

260

261

262

263

264

265

266

267

268

269

270

271

272

273

3.4 Silencing the ARK5 gene inhibits the proliferation and clonogenic ability of multidrug-resistant SGC7901/DDP cells

After the interference of the ARK5 gene, the number of cell clones in each group was tested by the colony formation assay under different drug treatments. The results showed that (Fig. 4 A, B), the colony formation rate of multidrug-resistant SGC7901/DDP cells with high ARK5 expression was significantly higher than that of parental SGC7901 cells. After silencing the ARK5 gene, the colony formation rate of SGC7901/DDP-shARK5 cells was significantly decreased.

\subsection{Silencing the ARK5 gene in multidrug-resistant GC7901/DDP cells increases chemotherapeutic drug-induced cell apoptosis.}

Flow cytometry was used to detect the apoptosis index of cells in each experimental group after chemotherapeutic drug treatment under the condition of ARK5 gene silencing. The results indicated that the apoptosis rate of multidrug-resistant SGC7901/DDP cells with high ARK5 expression was significantly lower than that of parental SGC7901 cells; after silencing the ARK5 gene, the apoptosis index of SGC7901/DDP-shARK5 cells was significantly increased (Fig. 5 A, B).

3.6 Silencing the ARK5 gene in multidrug-resistant SGC7901/DDP cells decreased the pump rate of Adriamycin in cells

Flow cytometry showed that the adriamycin pump rate of SGC7901/DDP cells with high ARK5 expression was significantly higher than that of SGC7901 cells with low ARK5 expression. However, after silencing the ARK5 gene, the adriamycin pump rate of SGC7901/DDP-shARK5 cells was decreased significantly compared with that of SGC7901/DDP cells (Fig. 6, Table 2).

\subsection{The DDP-induced multidrug-resistant cells with high ARK5 expression showed higher} resistance to 5-FU than the parental cells in vivo

Based on the results of tumor size from the subcutaneous dissection of mice (Fig. 7 A, B, C, D, E, F), after multiple administrations of 5-FU, the tumor volume inoculated with multidrug-resistant

SGC7901/DDP cells with higher expression levels of ARK5 gene was significantly larger than that of SGC7901 cells (Fig. 5 A, B). Thus, ARK5 gene expression is proportional to the resistance of gastric cancer cells to 5 -FU.

\section{Discussion}

The multidrug resistance of tumors is mainly manifested by the increased resistance and decreased sensitivity of tumor cells and is an indispensable factor influencing the chemotherapy effect in cancer patients. In this study, a series of experiments was conducted to preliminarily explore the relationship between the ARK5 gene and multidrug resistance of gastric cancer cells.

Primarily, western blotting showed that the expression level of ARK5 protein in drug-resistant SGC7901/DDP cells was much higher than that in parental SGC7901 cells, indicating that the drug resistance of cells was associated with ARK5 protein. Next, the cells in each group were treated with cisplatin (DDP), adriamycin (ADR), 5-fluorouracil (5-FU) and docetaxel (DR). Compared with SGC7901/DDP cells, the low survival rate and low $\mathrm{IC}_{50}$ index of SGC7901/DDP-shARK5 cells indicated 
274

275

276

277

278

279

280

281

282

283

284

285

286

287

288

289

290

291

292

293

294

295

296

297

298

299

300

301

302

303

304

305

306

307

308

309

310

311

312

313

314

that interfering with ARK5 gene expression could significantly enhance the sensitivity of cells to anticancer drugs, thus decreasing cell death. Meanwhile, the number of SGC7901/DDP-shARK5 colonies formed was small, indicating that interfering with ARK5 gene expression could significantly reduce the resistance of multidrug-resistant cells to chemotherapy drugs, thus inhibiting the proliferation activity and colony-forming efficiency of cells. The above results demonstrated that ARK5 plays an important role in maintaining the resistance of multidrug-resistant gastric cancer cells to chemotherapy drugs and could be effectively overcome by reducing ARK5 expression, thus reversing drug resistance. However, the mechanism remains unclear, and we continued to explore the molecular mechanism of ARK5 in drugresistant cells.

Increasing the active pump-out ability of anti-tumor drugs and reducing the concentration of the drugs in cells are known to be important ways for cancer cells to develop drug resistance, similar to the $\mathrm{ABC}$ transporter family member P-glycoprotein encoded by MDR1 and MDR2 acting as a pump to limit drug accumulation in cells to achieve drug resistance[14, 15, 41, 42]. In this study, we found that, after silencing the ARK5 gene in drug-resistant cells, the drug pump rate of cells to adriamycin was significantly reduced, indicating that the mechanism of action of ARK5 on multidrug-resistant cells was likely related to its inhibition of the active pump-out ability of chemotherapy drugs. Additionally, an abnormal apoptosis pathway can also induce the multidrug resistance of tumor cells. For example, the resistance of gastric cancer cells to anti-tumor drugs (DDP, 5-FU) was increased significantly after P53 gene mutation[43-45]. In our apoptosis experiments, we also found that silencing the ARK5 gene can significantly improve the apoptosis rate of drug-resistant cells after treatment with chemotherapy drugs. The above results showed that, at the cellular level, the ARK5 gene silencing effectively overcoming the resistance of drug-resistant cells to chemotherapy drugs is related to the reduction of cell resistance to apoptosis.

Additionally, the results from xenograft mouse models displayed that, after 5-FU treatment, the tumors of multidrug-resistant SGC7901/DDP cells with high ARK5 expression were significantly larger than those of SGC7901, indicating that the higher is the expression of ARK5, the higher is the drug resistance and survival of the tumor.

Notably, this experiment has so far confirmed the basic correlation of ARK5 and drug-resistance of gastric cancer cells, and this correlation is closely related to the active pump-out ability of the cells. Further research into this mechanism may be helpful to expand the clinical solutions to reduce multidrug resistance in gastric cancer cells.

In summary, based on the discoveries above, the specific signaling pathways and detailed mechanisms of the drug pumping rate and cell resistance to apoptosis by ARK5 need to be further studied.

\section{Conclusion}

Overall, this study verified that the ARK5 gene is closely related to the multidrug resistance of gastric cancer cells in vitro and in vivo, and ARK5 gene silencing could effectively reverse the resistance of multidrug-resistant gastric cancer cells to chemotherapeutic drugs. Moreover, this process is associated with the inhibition of the active pump-out ability of drug-resistant cells and the reduction of cell resistance to apoptosis by silencing the ARK5 gene. Finally, this study provides an experimental basis for the role of ARK5 in the multidrug resistance of gastric cancer cells.

Peer] reviewing PDF | (2020:02:45845:1:0:CHECK 14 May 2020) 
315

316

317

318

319

320

321

322

323

324

325

326

327

328

329

330

331

332

333

334

335

336

337

338

339

340

341

342

343

344

345

346

347

348

349

350

351

352

353

354

355

\section{Data Availability}

Interested readers can reproduce our results by using our algorithm.

\section{Authors' Contribution}

Hongtao Wan and Xiaowei Liu contributed equally to this work.

\section{References}

1. Frank-Stromborg, M., The epidemiology and primary prevention of gastric and esophageal cancer. A worldwide perspective. Cancer Nursing, 1989. 12(2): p. 53-64.

2. Karimi, P., et al., Gastric Cancer: Descriptive Epidemiology, Risk Factors, Screening, and Prevention. Cancer Epidemiology Biomarkers \& Prevention. 23(5): p. 700-713.

3. Ferlay, J., et al., Cancer incidence and mortality worldwide: Sources, methods and major patterns in GLOBOCAN 2012. International Journal of Cancer. 136(5): p. E359-E386.

4. Cornejo, C. and M. Portanova, [Comparative study of D1 and D2 ganglionic dissection in advanced gastric cancer at Rebagliati Hospital]. Rev Gastroenterol Peru, 2006. 26(4): p. 351-356.

5. Kang, J.H., et al., Salvage Chemotherapy for Pretreated Gastric Cancer: A Randomized Phase III Trial Comparing Chemotherapy Plus Best Supportive Care With Best Supportive Care Alone. Journal of Clinical Oncology Official Journal of the American Society of Clinical Oncology. 30(13): p. 1513-1518.

6. Cunningham, S.C., et al., Survival After Gastric Adenocarcinoma Resection: Eighteen-Year Experience at a Single Institution. Journal of Gastrointestinal Surgery, 2005. 9(5): p. 718-725.

7. Isobe, Y., et al., Gastric cancer treatment in Japan: 2008 annual report of the JGCA nationwide registry. Gastric Cancer, 2011. 14(4): p. 301-316.

8. Linn, S., et al., p53 and P-glycoprotein are often co-expressed and are associated with poor prognosis in breast cancer. British Journal of Cancer. 74(1): p. 63-68.

9. Zhang, X., et al., Alpha-tocopheryl succinate enhances doxorubicin-induced apoptosis in human gastric cancer cells via promotion of doxorubicin influx and suppression of doxorubicin efflux. Cancer Letters. 307(2): p. 0-181.

10. Gottesman, M.M., Biochemistry of Multidrug Resistance Mediated by the Multidrug Transporter. Annual Review of Biochemistry, 1993. 62(1): p. 385-427.

11. Chabner, B.A. and A. Fojo, Multidrug Resistance: P-glycoprotein and Its Allies--The Elusive Foes. Journal of the National Cancer Institute. 81(12): p. 910-913.

12. Biedler, J.L. and B.A. Spengler, Reverse transformation of multidrug-resistant Cells. Cancer \& Metastasis Reviews, 1994. 13(2): p. 191-207.

13. C, C.-C., et al., Expression of the multidrug resistance gene product (P-glycoprotein) in human normal and tumor tissues. The journal of histochemistry and cytochemistry : official journal of the Histochemistry Society, 1990. 38(9): p. 1277-87.

14. Wu, C.P. and S. V. Ambudkar, The pharmacological impact of ATP-binding cassette drug transporters on vemurafenib-based therapy. Acta Pharmaceutica Sinica B. 4(2): p. 105-111.

15. $\mathrm{X}, \mathrm{X}$. and $\mathrm{L} . \mathrm{XJ}$, Overcoming drug efflux-based multidrug resistance in cancer with nanotechnology. Chinese journal of cancer, 2012. 31(2): p. 100-9.

16. Goldstein, L.J., et al., Expression of Multidrug Resistance Gene in Human Cancers. J Natl Cancer Inst. 81(2): p. 116-124.

Peer) reviewing PDF | (2020:02:45845:1:0:CHECK 14 May 2020) 
356

357

358

359

360

361

362

363

364

365

366

367

368

369

370

371

372

373

374

375

376

377

378

379

380

381

382

383

384

385

386

387

388

389

390

391

392

393

394

395

396

17. D, Z. and F. D, New insights into the mechanisms of gastric cancer multidrug resistance and future perspectives. Future oncology (London, England), 2010. 6(4): p. 527-37.

18. Gürel, S., et al., High Expression of Multidrug Resistance-1 (MDR-1) and its Relationship with Multiple Prognostic Factors in Gastric Carcinomas in Patients in Turkey. Journal of International Medical Research, 1999. 27(2): p. 79-84. 19. $\mathrm{JH}, \mathrm{C}$., et al., Expression of multidrug resistance-associated protein1,P-glycoprotein, and thymidylate synthase in gastric cancer patients treated with 5-fluorouracil and doxorubicin-based adjuvant chemotherapy after curative resection. British journal of cancer, 2002. 86(10): p. 1578-85.

20. Fan, K.C., et al., Expression of multidrug resistance-related markers in gastric cancer. Anticancer Research, 2000. 20(6C): p. 4809-4814.

21. CC, L., et al., Metformin triggers the intrinsic apoptotic response in human AGS gastric adenocarcinoma cells by activating AMPK and suppressing mTOR/AKT signaling. International journal of oncology, 2019. 54(4): p. 1271-1281. 22. Hardie, D.G., AMP-activated protein kinase-an energy sensor that regulates all aspects of cell function. 2011. 25(18): p. 1895-1908.

23. HW, C., et al., Knockdown of B-catenin controls both apoptotic and autophagic cell death through LKB1/AMPK signaling in head and neck squamous cell carcinoma cell lines. Cellular signalling, 2013. 25(4): p. 839-47.

24. Yu, Z., et al., Salinomycin enhances doxorubicin sensitivity through reversing the epithelial-mesenchymal transition of cholangiocarcinoma cells by regulating ARK5. Brazilian Journal of Medical \& Biological Research, 2017. 50(10).

25. $\mathrm{Xu}, \mathrm{T}$. , et al., ARK5 promotes doxorubicin resistance in hepatocellular carcinoma via epithelial-mesenchymal transition. Cancer Letters: p. S0304383516302610.

26. Liu, J., et al., Expression level of NUAK1 in human nasopharyngeal carcinoma and its prognostic significance. European Archives of Oto-Rhino-Laryngology.

27. Suzuki, A., et al., ARK5 Is a Tumor Invasion-Associated Factor Downstream of Akt Signaling. Molecular \& Cellular Biology, 2004. 24(8): p. 3526-3535.

28. SA, H., et al., Complexes between the LKB1 tumor suppressor, STRAD alpha/beta and MO25 alpha/beta are upstream kinases in the AMP-activated protein kinase cascade. Journal of biology, 2003. 2(4): p. 28.

29. M, M., H. SP, and C. M, Mammalian TAK1 activates Snf1 protein kinase in yeast and phosphorylates AMPactivated protein kinase in vitro. The Journal of biological chemistry, 2006. 281(35): p. 25336-43.

30. Deregulated MYC expression induces dependence upon AMPK-related kinase 5. Nature. 483(7391): p. 608-612. 31. Li, M., et al., Inhibition of AMPK-related kinase 5 (ARK5) enhances cisplatin cytotoxicity in non-small cell lung cancer cells through regulation of epithelial-mesenchymal transition. American Journal of Translational Research, 2017. 9(4): p. 1708-1719.

32. Chen, D., et al., Knockdown of ARK5 Expression Suppresses Invasion and Metastasis of Gastric Cancer. Cellular Physiology \& Biochemistry, 2017. 42(3): p. 1025-1036.

33. Friedman, E.A., New chemotherapeutic drug sensitivity for colon carcinomas in monolayer culture. Cancer Research, 1988. 48(11): p. 3236-3244.

34. V, B., et al., Lipodox ${ }^{\circledR}$ (generic doxorubicin hydrochloride liposome injection): in vivo efficacy and bioequivalence versus Caelyx ${ }^{\circledast}$ (doxorubicin hydrochloride liposome injection) in human mammary carcinoma (MX-1) xenograft and syngeneic fibrosarcoma (WEHI 164) mouse models. BMC cancer, 2017. 17(1): p. 405.

35. CL, D., et al., Tetrandrine achieved plasma concentrations capable of reversing MDR in vitro and had no apparent effect on doxorubicin pharmacokinetics in mice. Cancer chemotherapy and pharmacology, 2007. 60(5): p. 
397

398

399

400

401

402

403

404

405

406

407

408

409

410

411

412

413

414

415

416

417

418
741-50.

36. 张慧, KLF8 在缺氧诱导胃癌细胞多药耐药中的作用及机制研究. 2013，第四军医大学. In Chinese.

37. Yue, Z., et al., Salinomycin decreases doxorubicin resistance in hepatocellular carcinoma cells by inhibiting the \&\#x3B2;-catenin/TCF complex association via FOXO3a activation. Oncotarget. 6(12).

38. Wang, X., Preliminary Study on Preparation and Pharmaceutic Features of Adrimycin-loaded Human Serum Albumin Microspheres. Journal of Sichuan University, 2004. 35(1): p. 107.

39. M?hler, M., New Perspectives in the Treatment of Advanced Gastric Cancer: S-1 as a Novel Oral 5-FU Therapy in Combination with Cisplatin. Chemotherapy: p. 62-70.

40. Von Kalle, C., et al., Growth and metastasis of tumor cells isolated from a human renal cell carcinoma implanted into different organs of nude mice. 1986. 46(8): p. 4109.

41. C, N., et al., Modulation of the classical multidrug resistance (MDR) phenotype by RNA interference (RNAi). FEBS letters, 2003. 545(null): p. 144-50.

42. EA, A., et al., MRP1 expression in CTCS confers resistance to irinotecan-based chemotherapy in metastatic colorectal cancer. International journal of cancer, 2016. 139(4): p. 890-8.

43. V, M. and P. L, Involvement of $p 53$ in the repair of DNA double strand breaks: multifaceted Roles of $p 53$ in homologous recombination repair (HRR) and non-homologous end joining (NHEJ). Sub-cellular biochemistry, 2014. 85(undefined): p. 321-36.

44. N, M., et al., The evaluation of gastric cancer sensitivity to 5-FU/CDDP in terms of induction of apoptosis: timeand $p 53$ expression-dependency of anti-cancer drugs. Oncology reports, 2005. 14(3): p. 609-15.

45. S, C., et al., Expression of p53 protein and resistance to preoperative chemotherapy in locally advanced gastric carcinoma. Cancer, 1998. 83(9): p. 1917-22. 


\section{Table $\mathbf{1}$ (on next page)}

Drug sensitivity of SGC7901, SGC7901/DDP, SGC7901/DDP-shARK5 and SGC7901/DDPNC cells.

The data are expressed as the means $\pm \mathrm{SEM} ; \mathrm{n}=3 ;{ }^{* *} \mathrm{P}<0.01$ versus $\mathrm{SGC7901;} \# \# \mathrm{P}<0.01$ versus SGC7901/DDP. 


\begin{tabular}{lllll}
\hline \multirow{2}{*}{ Groups } & \multicolumn{4}{c}{ IC $_{\mathbf{5 0}}$ value $(\boldsymbol{\mu g} / \mathbf{m l})$} \\
\cline { 2 - 5 } & \multicolumn{1}{c}{ DDP } & \multicolumn{1}{c}{$\mathbf{5 F u}$} & \multicolumn{1}{c}{ ADR } & \multicolumn{1}{c}{ DR } \\
\hline SGC7901 & $1.66 \pm 0.14$ & $5.12 \pm 0.38$ & $0.38 \pm 0.06$ & $0.036 \pm 0.007$ \\
SGC7901/DDP & $6.54 \pm 0.36^{* *}$ & $34.28 \pm 2.35^{* *}$ & $1.58 \pm 0.18^{* *}$ & $0.128 \pm 0.016^{* *}$ \\
SGC7901/DDP-shARK5 & $1.42 \pm 0.09^{\# \#}$ & $4.92 \pm 0.28^{\# \#}$ & $0.32 \pm 0.09^{\# \#}$ & $0.031 \pm 0.009^{\# \#}$ \\
SGC7901/DDP-NC & $7.12 \pm 0.42$ & $32.64 \pm 3.42$ & $1.42 \pm 0.12$ & $0.138 \pm 0.012$ \\
\hline
\end{tabular}

1 


\section{Table 2 (on next page)}

Accumulation and retention of doxorubicin in gastric cancer cells

The drug pump rates of SGC7901, SGC7901/DDP, SGC7901/DDP-shARK5 and SGC7901/DDPNC cells were calculated using the following formula: pump rate of $A D R=$ (intracellular ADR accumulation - intracellular ADR retention)/(intracellular ADR accumulation) $\times 100 \%$. The data are expressed as the means $\pm \mathrm{SEM} ; \mathrm{n}=3 ; * * \mathrm{P}<0.01$ versus $\mathrm{SGC7901;} \# \# \mathrm{P}<0.01$ versus SGC7901/DDP. 


\begin{tabular}{llll}
\hline \multirow{2}{*}{ Groups } & \multicolumn{2}{c}{ Fluorescence Intensity } & \multirow{2}{*}{ Drug pump rate } \\
\cline { 2 - 4 } & Accumulation & Retention & \\
\hline SGC7901 & $1.913 \pm 0.086$ & $1.864 \pm 0.082$ & $0.026 \pm 0.008$ \\
SGC7901/DDP & $2.564 \pm 0.184$ & $1.713 \pm 0.078$ & $0.332 \pm 0.028^{* *}$ \\
SGC7901/DDP-shARK5 & $2.748 \pm 0.092$ & $2.701 \pm 0.096$ & \\
SGC7901/DDP-NC & $3.245 \pm 0.204$ & $2.264 \pm 0.172$ & $0.017 \pm 0.008^{\# \#}$ \\
& & & $0.302 \pm 0.022$ \\
\hline
\end{tabular}

1 


\section{Figure 1}

ARK5 expression levels in parental and multidrug-resistant cell lines.

In this baseline expression level experiment, the protein expression level of ARK5 in SGC7901/DDP was significantly higher than that of SGC7901. The values in a representative blot are shown as the means \pm SEM $(n=3 ; * * P<0.01$ versus SGC7901). 


\section{SGC7901 SGC7901/DDP}
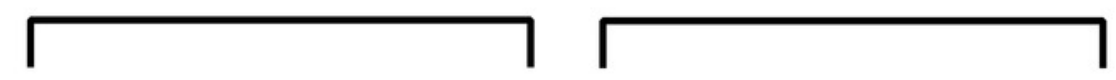

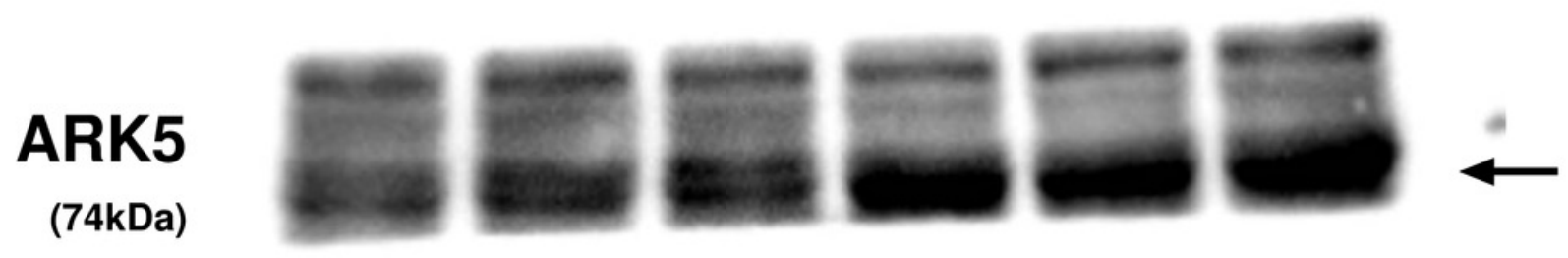

$\beta$-actin

(43kDa)

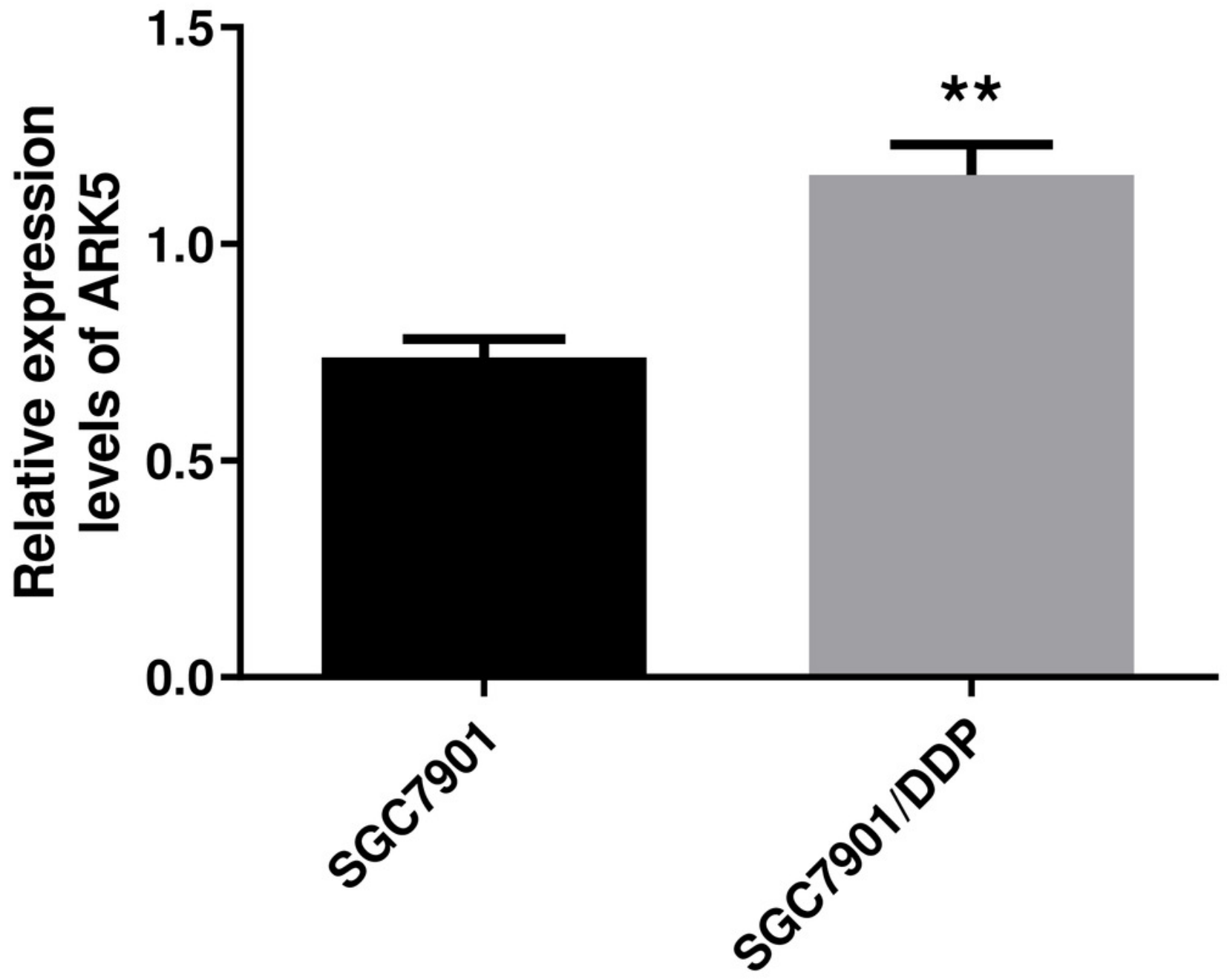




\section{Figure 2}

western blot analysis of the interference efficiency of LV-ARK5-RNAi.

The differential expression levels of ARK5 in SGC7901, SGC7901/DDP, SGC7901/DDP-shARK5, SGC7901/DDP-NC cells are shown as the means \pm SEM $(n=3 ; \# \# P<0.01$ versus SGC7901/DDP; ${ }^{* * P}<0.01$ versus SGC7901). ARK5 expression in SGC7901/DDP-shARK5 was comparable to that of the baseline expression of the parental SGC7901 cell line. 


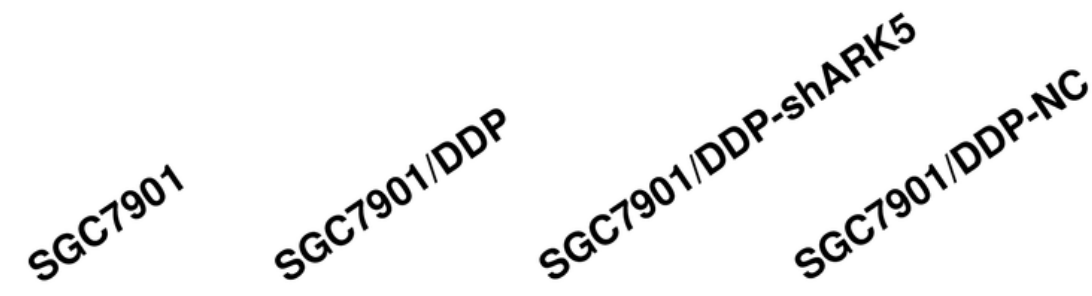

ARK5

(74kDa)

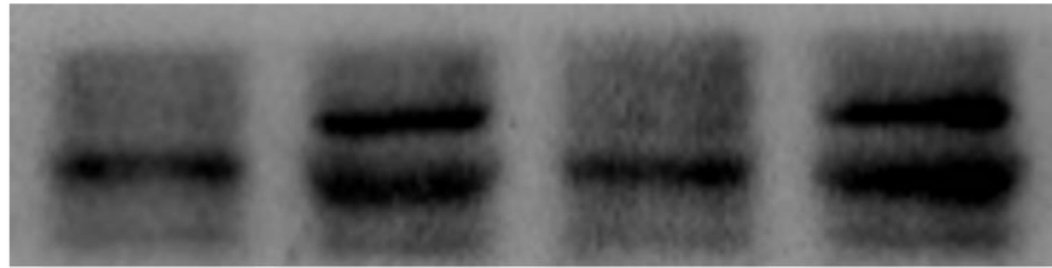

$\beta$-actin

(43kDa)

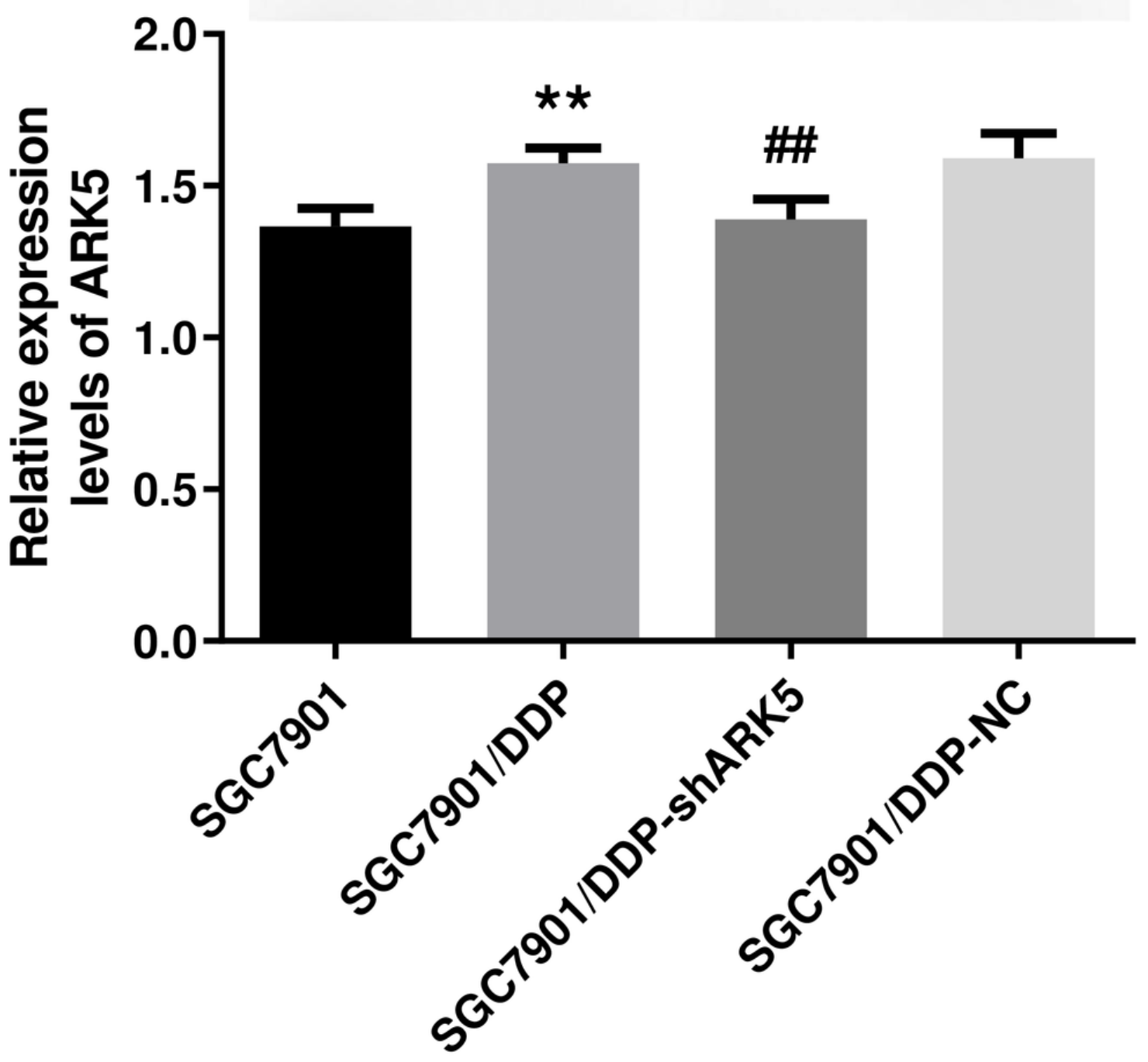


Figure 3

Effects of ARK5 gene Silencing on the survival rates in multidrug-resistant gastric cancer cells.

After treatment with the four anti-neoplastic drugs (DDP, 5-Fu, ADR and DR), the survival rates of SGC7901, SGC7901/DDP, SGC7901/DDP-shARK5 and SGC7901/DDP-NC cells were measured by the CCK-8 assay. The values are presented as the means \pm SEM $(n=3$; **P<0.01 versus SGC7901; \#\#P<0.01 versus SGC7901/DDP).
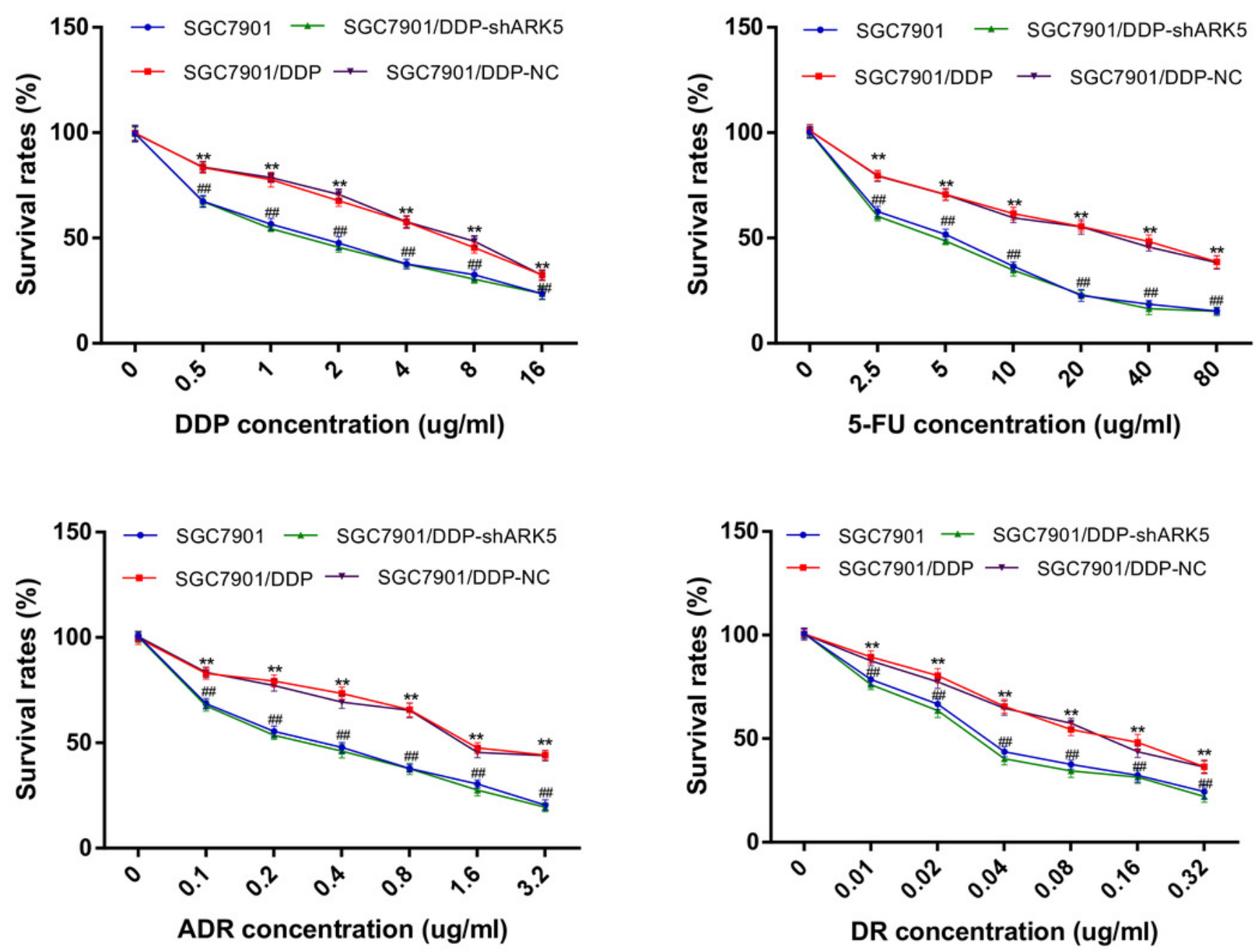
Figure 4 (on next page)

Effects of ARK5 gene Silencing on the colony-forming ability in multidrug-resistant gastric cancer cells.

The four anti-tumor drugs DDP, 5-FU, ADR, DR were used to treat each experimental group according to the peak serum concentrations $(2.0 \mu \mathrm{g} / \mathrm{ml}, 10 \mu \mathrm{g} / \mathrm{ml}, 0.4 \mu \mathrm{g} / \mathrm{ml}$ and $0.04 \mu \mathrm{g} / \mathrm{ml}$, respectively). (A) The colony formation assays of SGC7901, SGC7901/DDP, SGC7901/DDPShARK5 and SGC7901/DDP-NC cells are shown on the plates. (B) The rates of colony formation were measured after treatment with DDP, 5-Fu, ADR and DR. The data are

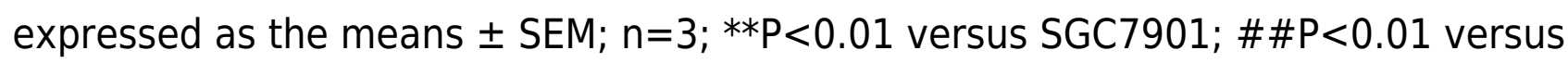
SGC7901/DDP. 


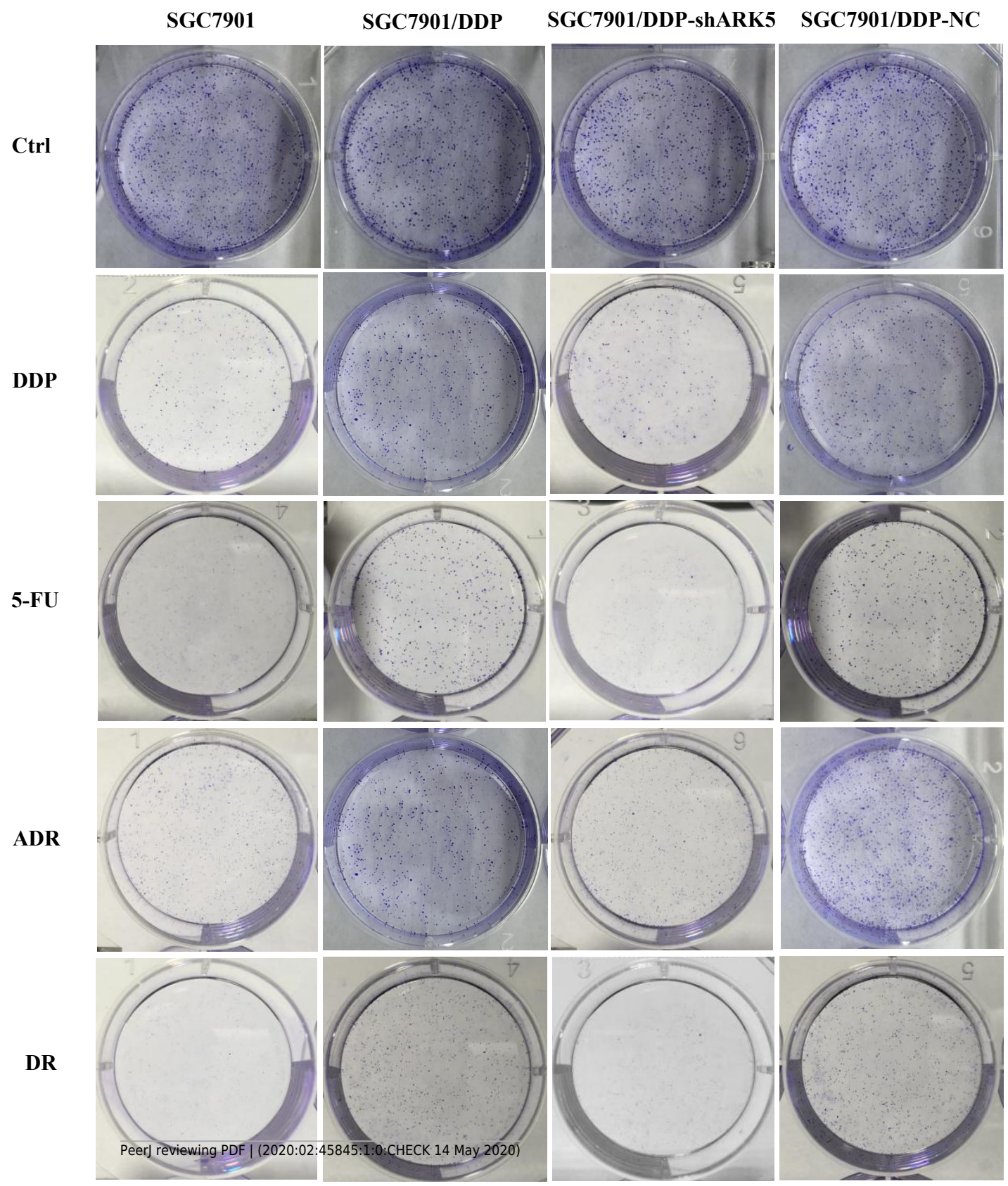




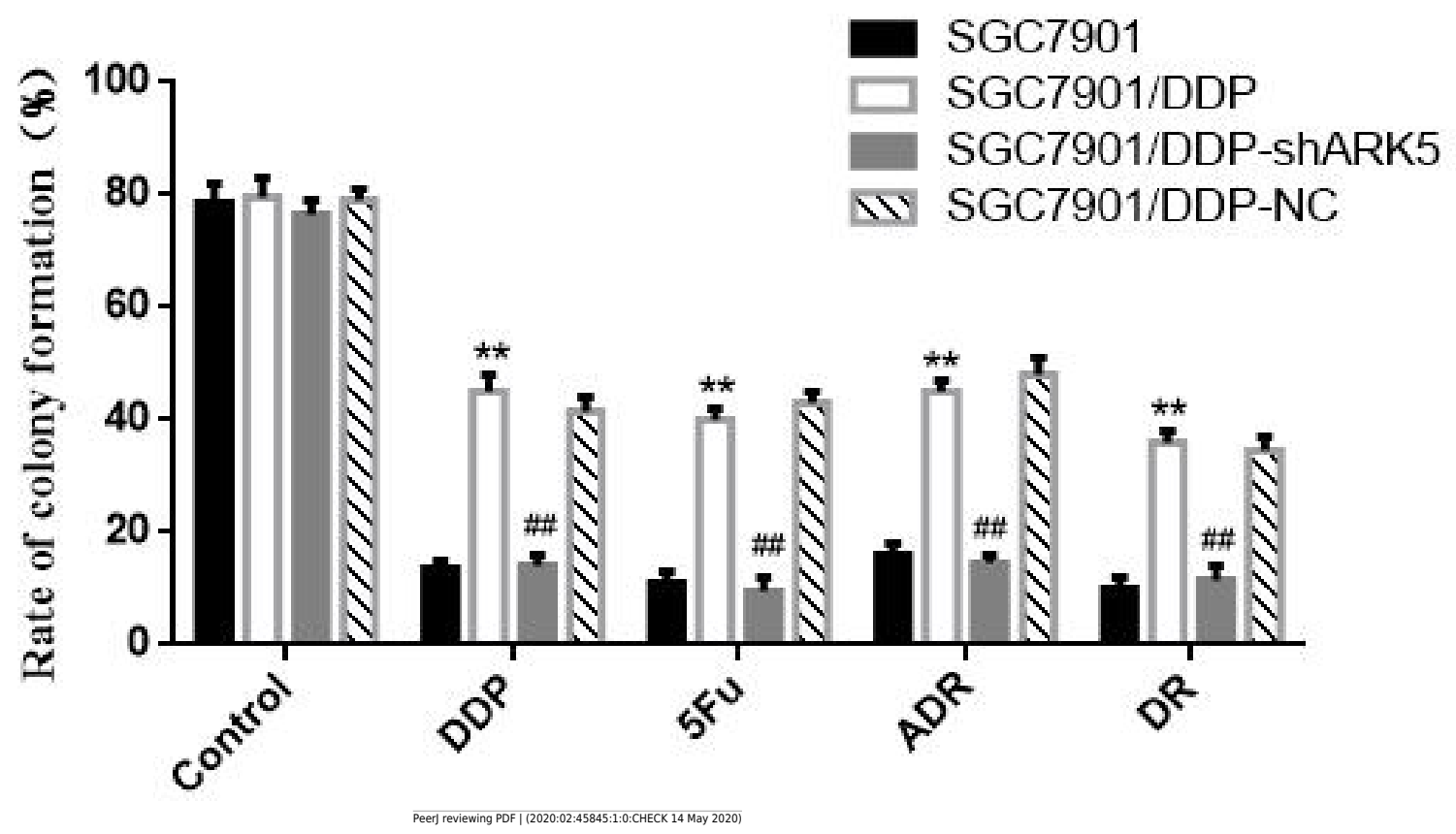


Figure $\mathbf{5}$ (on next page)

Effects of ARK5 gene silencing on the apoptosis rate in SGC7901 cells.

The concentrations of the four drugs DDP, 5-FU, ADR and DR used to treat each group were $2.0 \mu \mathrm{g} / \mathrm{ml}, 10 \mu \mathrm{g} / \mathrm{ml}, 0.4 \mu \mathrm{g} / \mathrm{ml}$ and $0.04 \mu \mathrm{g} / \mathrm{ml}$ respectively. (A) The apoptosis rates of SGC7901, SGC7901/DDP, SGC7901/DDP-shARK5 and SGC7901/DDP-NC cells were measured after treatment with DDP, 5-Fu, ADR or DR. The lower left quadrant indicates viable cells, and the other three quadrants represent cells with varying degrees of apoptosis. (B) Bar graph of the apoptosis rate analysis. The data are expressed as the means $\pm S E M ; n=3 ; * * P<0.01$ versus SGC7901; \#\#P<0.01 versus SGC7901/DDP. 
SGC7901
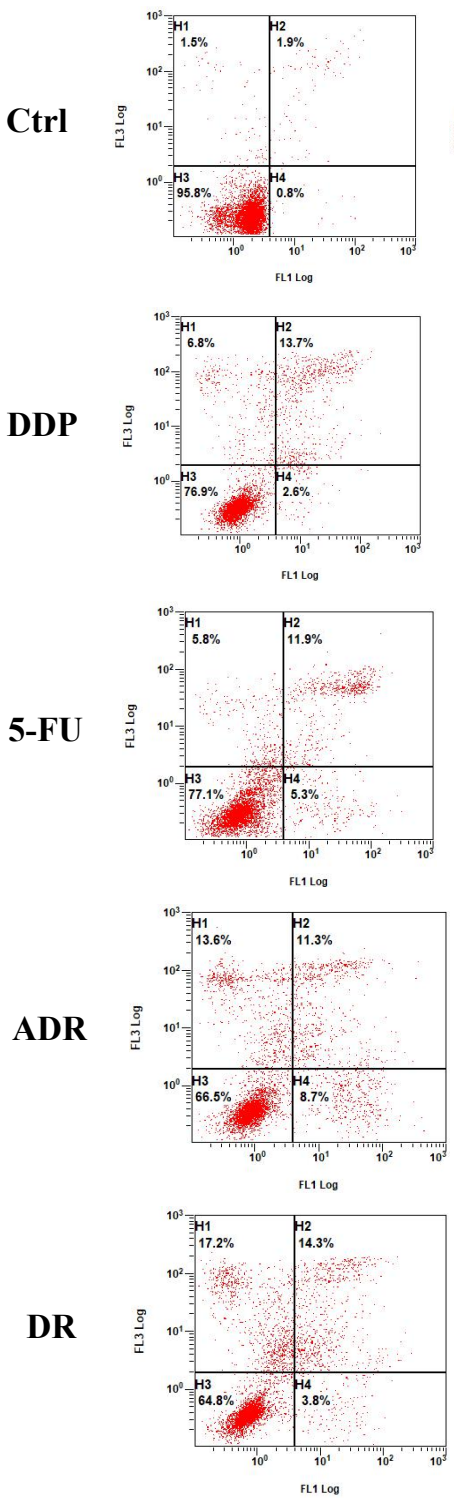

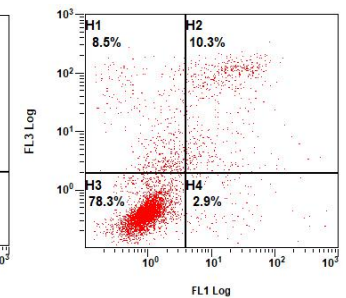

\section{SGC7901/DDP}
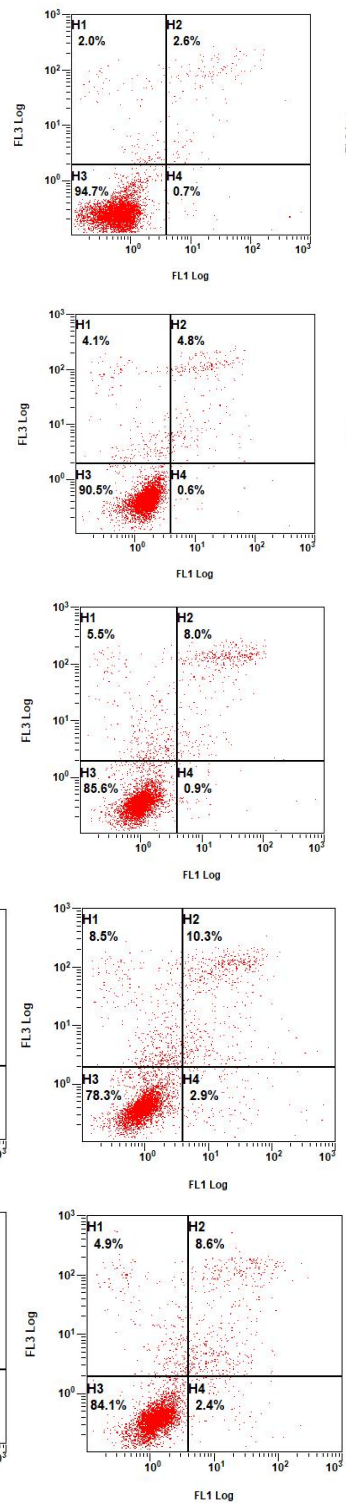

SGC7901/DDP-shARK5

SGC7901/DDP-NC
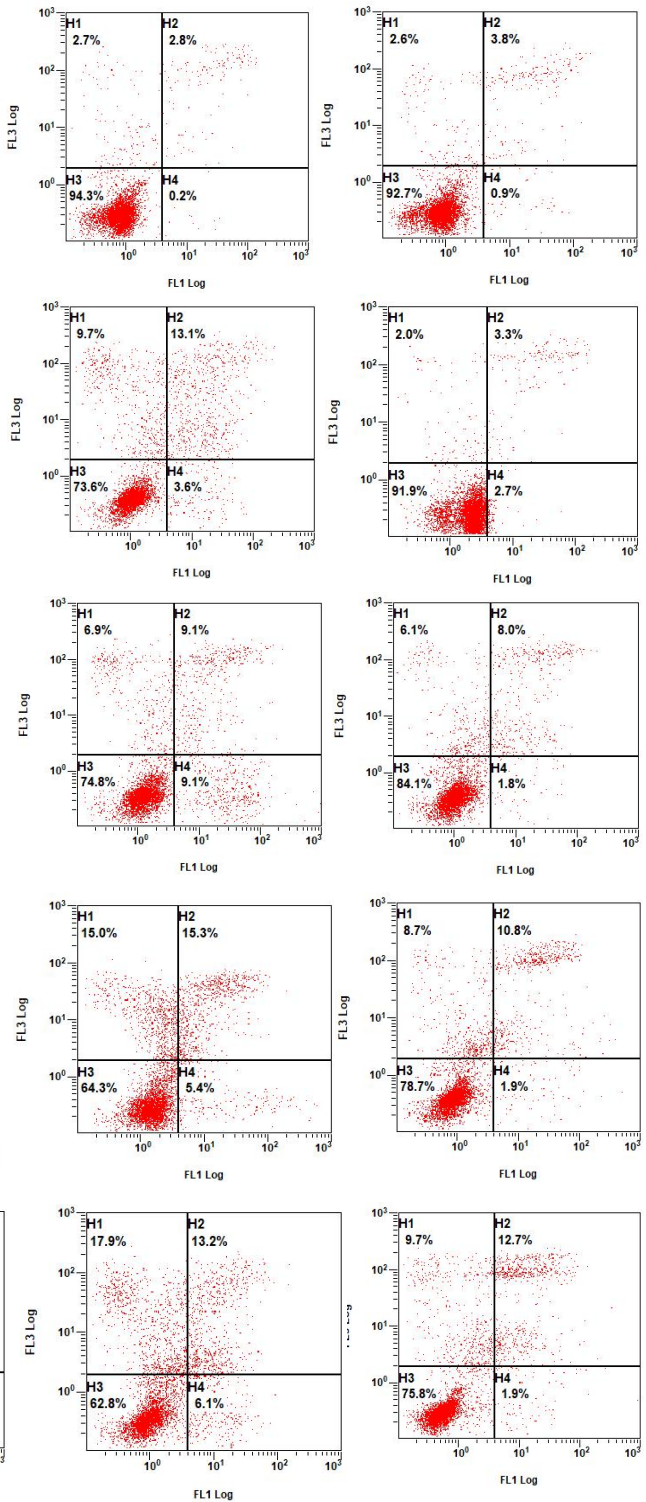


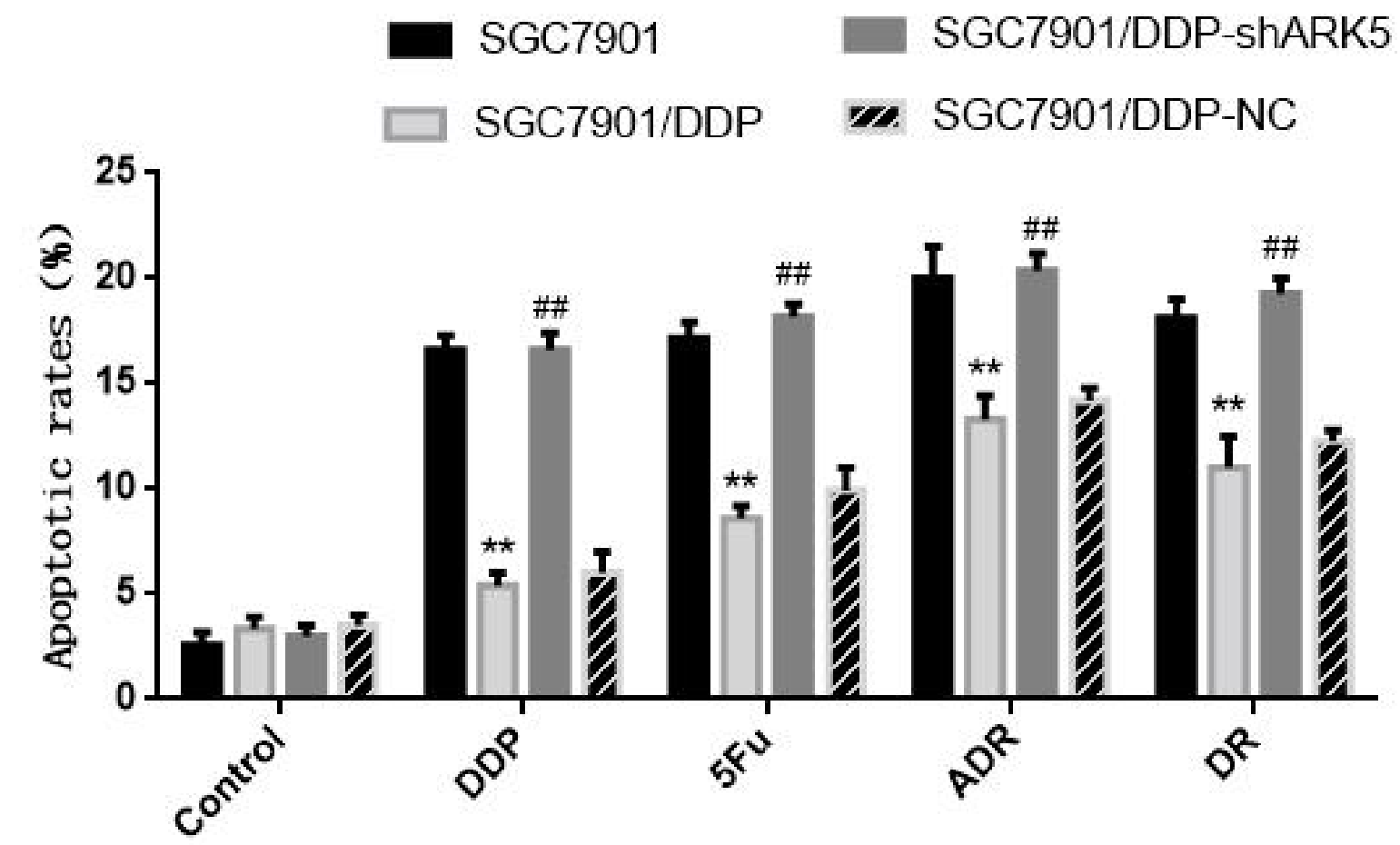


Figure 6

Effects of ARK5 gene silencing on the adriamycin pump rate in SGC7901 cells.

After cells were treated with $5 \mu \mathrm{g} / \mathrm{ml}$ of adriamycin, the ADR accumulation and retention of SGC7901, SGC7901/DDP, SGC7901/DDP-shARK5 and SGC7901/DDP-NC cells were measured by flow cytometry. 

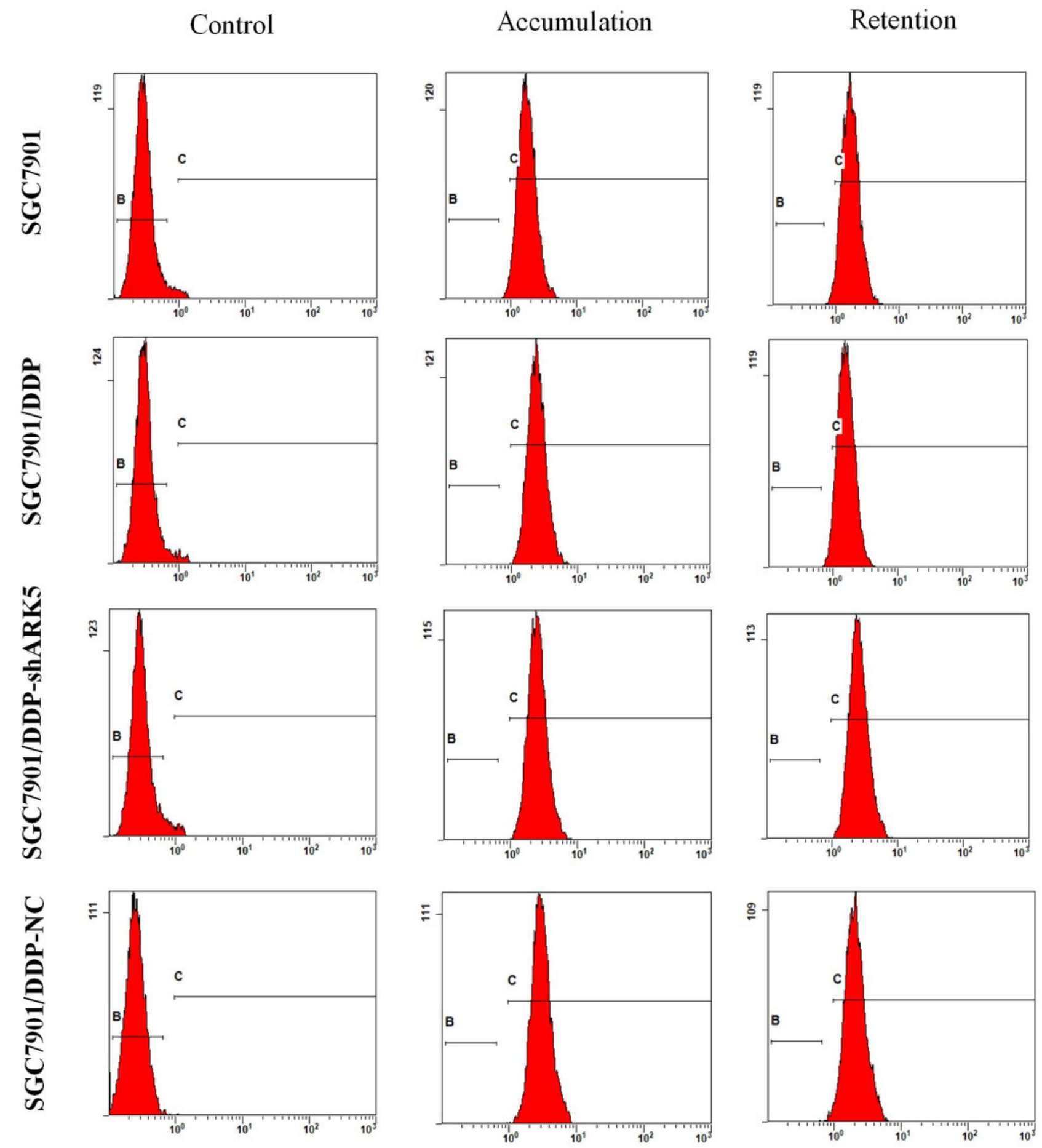


\section{Figure 7 (on next page)}

Effects of the multidrug resistance of gastric cancer cells on the tumor volume in mice treated with the chemotherapeutic drug 5-FU.

To further confirm that gastric cancer cells with high ARK5 expression have a higher resistance to chemotherapeutic drugs, we used the representative chemotherapeutic drug 5FU to conduct in vivo experiments in mice and then administered 5-FU at a concentration of $20 \mathrm{mg} / \mathrm{kg}$ twice a week. (A) Sacrificed and depilated NOD/SCID mice. (B) Body weight variation of mice throughout the 31-day experiment. (C) Dissected tumors from mice. (D) Quantification of the tumor weight of mice in the SGC7901 and SGC7901/DDP groups. (E) Quantification of the tumor volume of mice in the SGC7901 and SGC7901/DDP groups. (F) Tumor growth curves of the two groups of mice. The data are expressed as the means \pm SEM, $n=5 ; * * p<0.01$ versus SGC7901; \#\#P<0.01 versus SGC7901. 


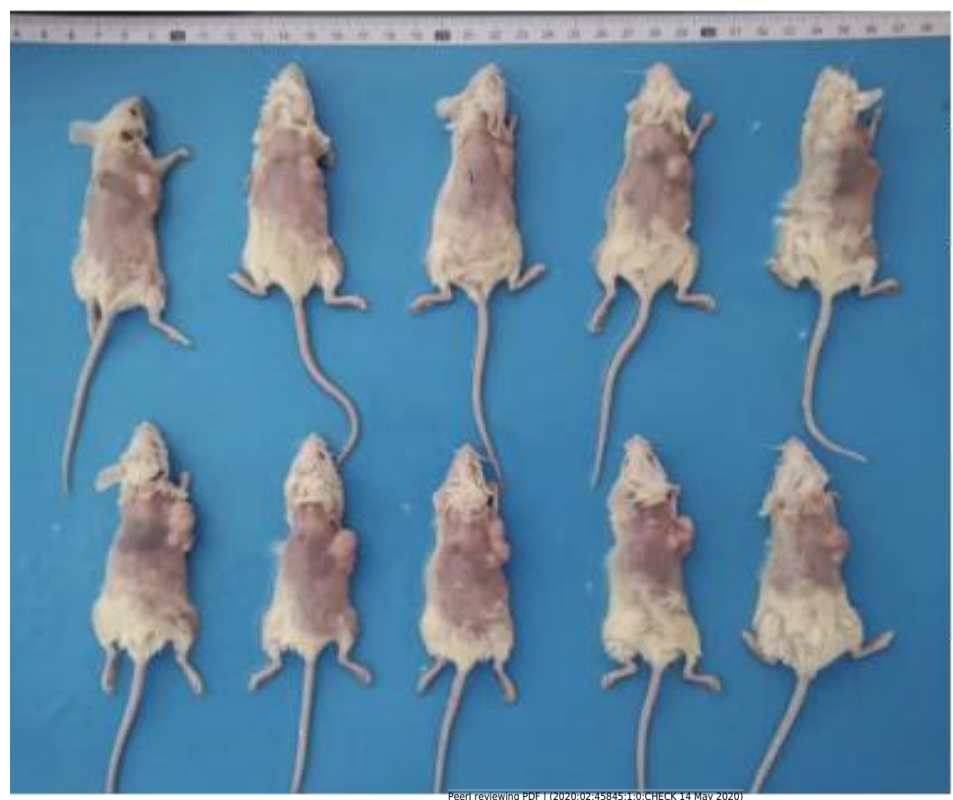

SGC7901

SGC7901/DDP 
B

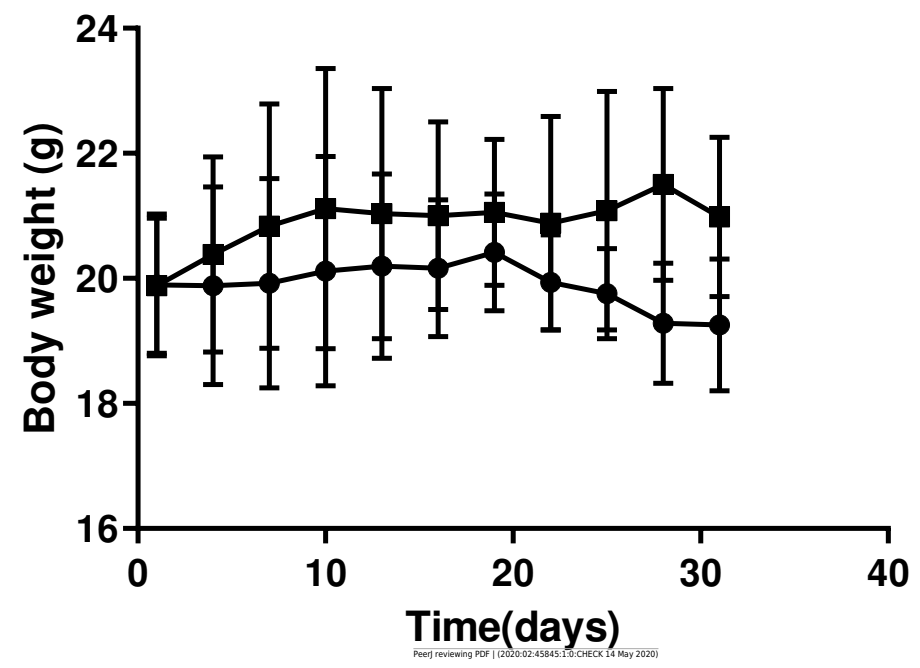

- SGC7901

- SGC7901/DDP 
C

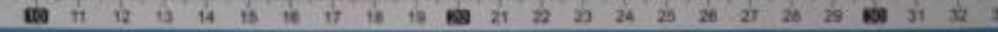

SGC7901

SGC7901/DDP 


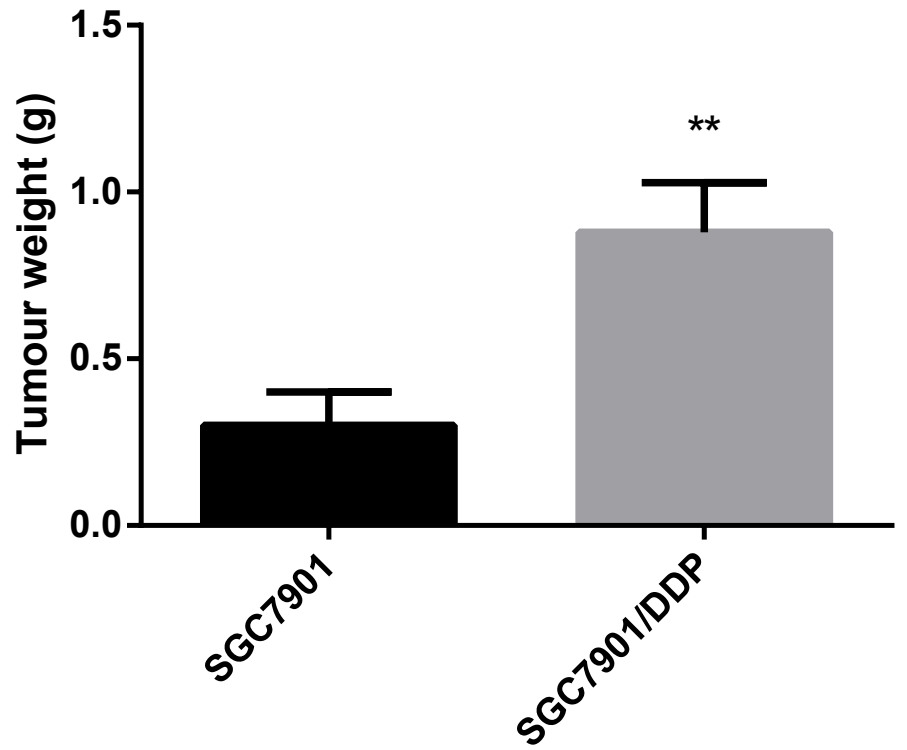


E

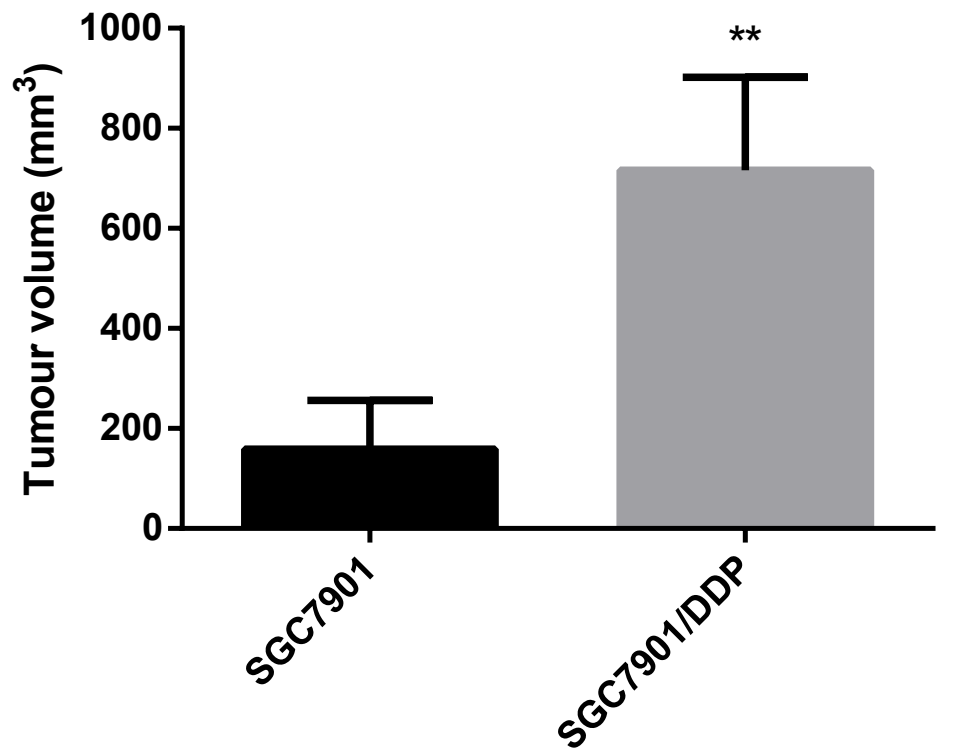


$\mathbf{F}$

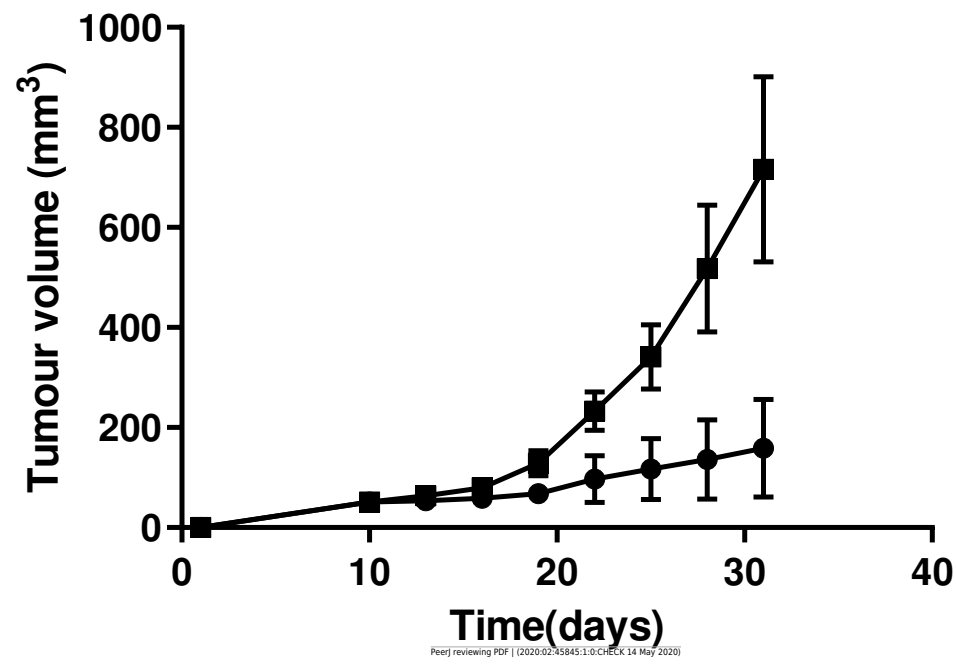

- SGC7901

$\Rightarrow$ SGC7901/DDP 